\title{
Signal Processing Algorithms for Down-Stream Traffic in Next Generation 10 Gbit/s Fixed-Grid Passive Optical Networks
}

\author{
Rameez Asif, Rabeea Basir, and Ramshah Ahmad \\ Telecommunication Engineering Department, University of Engineering and Technology (UET), Taxila 47050, Pakistan \\ Correspondence should be addressed to Rameez Asif; dr.rameezasif@outlook.com
}

Received 28 March 2014; Revised 20 May 2014; Accepted 3 June 2014; Published 22 June 2014

Academic Editor: Jayanta K. Sahu

Copyright (C) 2014 Rameez Asif et al. This is an open access article distributed under the Creative Commons Attribution License, which permits unrestricted use, distribution, and reproduction in any medium, provided the original work is properly cited.

\begin{abstract}
We have analyzed the impact of digital and optical signal processing algorithms, that is, Volterra equalization (VE), digital backpropagation (BP), and optical phase conjugation with nonlinearity module (OPC-NM), in next generation $10 \mathrm{Gbit} / \mathrm{s}$ (also referred to as XG) DP-QPSK long haul WDM (fixed-grid) passive optical network (PON) without midspan repeaters over $120 \mathrm{~km}$ standard single mode fiber (SMF) link for downstream signals. Due to the compensation of optical Kerr effects, the sensitivity penalty is improved by $2 \mathrm{~dB}$ by implementing BP algorithm, $1.5 \mathrm{~dB}$ by VE algorithm, and $2.69 \mathrm{~dB}$ by OPC-NM. Moreover, with the implementation of NL equalization technique, we are able to get the transmission distance of $126.6 \mathrm{~km}$ SMF for the $1: 1024$ split ratio at $5 \mathrm{GHz}$ channel spacing in the nonlinear region.
\end{abstract}

\section{Introduction}

Due to the increasing demand of bandwidth and capacity requirements from enterprises and households, the data rates of broadband access network will be required over $10 \mathrm{Gbit} / \mathrm{s}$ for each customer. Several passive optical network (PON) architectures have been proposed, that is, G-PON, E-PON, TDM-PON, and so forth, in order to remove the capacity bottleneck. Recently, the $10 \mathrm{Gbit} / \mathrm{s}$ long haul wavelength-division multiplexed- (WDM-) PON system has been demonstrated with coherent detection; this configuration represents a significant improvement with a receiver sensitivity of $-45 \mathrm{dBm}$ (25 photons/bit) [1].

With the implementation of advanced modulation formats, that is, QPSK, QAM, and so forth, and multiplexing techniques, that is, dual-polarization and so forth, the system performance is limited due to fiber linear and nonlinear effects [2]. These effects are very much dominant at higher signal launch powers and in WDM systems with narrow channel spacing [3]. Rosenkranz and von Hoyningen-Huene presented the results of nonlinearity compensation in access networks; however, the results are limited to the transmitters where optical field is derived from the modulation current with the directly modulated laser (DML) rate equations; thus modulation nonlinearity and chirp are included [4] and are detected by direct detection method. In this paper, we have numerically analysed the transmission characteristics and the nonlinear equalization techniques by employing $\mathrm{BP}$, VE, and OPC-NM in $10 \mathrm{Gbit} / \mathrm{s}$ DP-QPSK long haul WDMPON transmission with coherent receivers, for downstream signals. Furthermore; the impact of nonlinear equalization algorithm on the transmission distance and split ratio factor is investigated.

\section{Nonlinear Equalization Methods}

In this section, we will briefly discuss the nonlinear equalization techniques implemented in this paper.

2.1. Digital Backpropagation (BP). The joint compensation of linear and nonlinear transmission impairments is implemented by inversely solving the nonlinear Schrodinger equation (NLSE), as in (1). This method is termed as digital backpropagation (BP) [2, 5-12], and it is a topic of high interest in recent years. We have implemented $\mathrm{BP}$ algorithm by using the 
simplest symmetric split-step Fourier method (SSFM) with constant step-size method [7], as in (2). Consider

$$
\begin{gathered}
\frac{\partial E}{\partial z}=(-\widehat{N}-\widehat{D}) E \\
E(z+h, t)=\exp \left(\frac{h \widehat{D}}{2}\right) \exp (h \widehat{N}) \exp \left(\frac{h \widehat{D}}{2}\right) \cdot E(z, t),
\end{gathered}
$$

whereas $\widehat{D}$ and $\widehat{N}$ are the linear and nonlinear operators, respectively, to solve the inverse NLSE.

2.2. Volterra Equalization (VE). Another alternative approach for joint compensation of linear and nonlinear effects is the Volterra equalization (VE) method $[4,13]$, as in Figure 1. This method is an expansion of a linear feed forward equalizer (FFE) and decision feedback equalizer (DFE) of higherorder combinations of the delayed signal. The joint FFEDFE algorithm is applied in order to process the I- and Q-tributaries to avoid intersymbol-interference (ISI). The optimal coefficients are calculated for the equalizer according to the minimum mean squared error (MMSE) criterion and are given by the well-known Wiener solution for the joint FFE-DFE algorithm [14].

These two methods, that is, BP and VE, are the postprocessing methodologies implemented with coherent receivers. We have also implemented an all-optical signal preprocessing method for nonlinear equalization, which is termed as optical phase conjugation with nonlinearity module (OPC-NM) [15].

2.3. All-Optical Signal Preprocessing. This module, as in Figure 2, contains highly nonlinear fiber (HNLF) and optical phase conjugation (OPC) module $[15,16]$. The nonlinear stage is implemented by $19 \mathrm{~km}$ of SMF with input launch power $P_{m}$ tuned by an erbium doped fiber amplifier (EDFA). The OPC stage uses the four-wave mixing of the signal with a $\mathrm{CW}$ laser as the pump in a $120 \mathrm{~m}$ long HNLF. The generated signal is passed through band-pass filter, amplified, and launched into the passive fiber link. The parameters of this module, that is, fiber length $L_{m}$, signal power $P_{m}$, and nonlinear coefficient $\gamma_{m}$, are adjusted along with OPC module so that it produces negative nonlinear phase shift of $\left(-\Delta \phi_{m}\right)$, canceling the original $\left(\Delta \phi_{m}\right)$ from fiber transmission link.

\section{Numerical Model}

The architecture, as in Figure 3, consists of optical line terminal (OLT) having $3 \mathrm{CW}$ lasers, which are individually modulated with DP-QPSK signal and multiplexed together (with $50 \mathrm{GHz}, 10 \mathrm{GHz}$, and $5 \mathrm{GHz}$ channel spacing) resulting in $10 \mathrm{Gbit} / \mathrm{s}$ WDM DP-QPSK signal per wavelength. The applied pattern was a pseudorandom bit sequence (PRBS) of length $2^{15}-1$ and RZ pulse shaping is used. An EDFA at the OLT adjusts the signal input launch power into the fiber backhaul and passive optical splitters are used to distribute the signal to the optical network units (ONUs). Moreover, narrow optical filtering is used at the transmitter to compensate the spectral overlapping. The standard single mode fiber is used

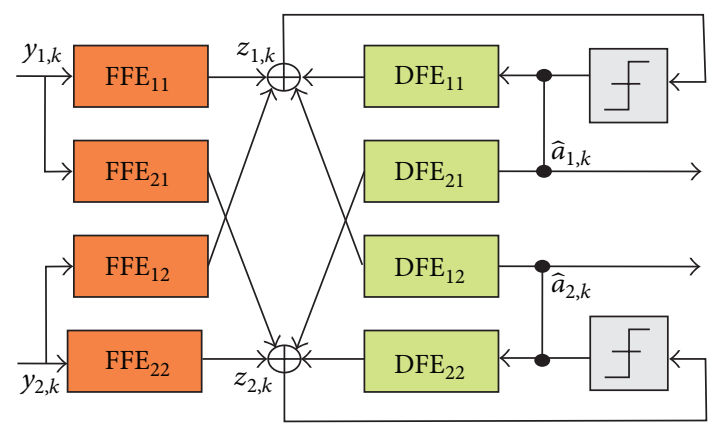

FIGURE 1: Numerical model of Volterra-based nonlinear FFE-DFE equalizer.

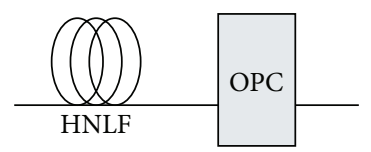

FIGURE 2: Basic architecture of optical phase conjugation with nonlinearity module (OPC-NM).

Optical line termination (OLT) Optical network unit (ONU)

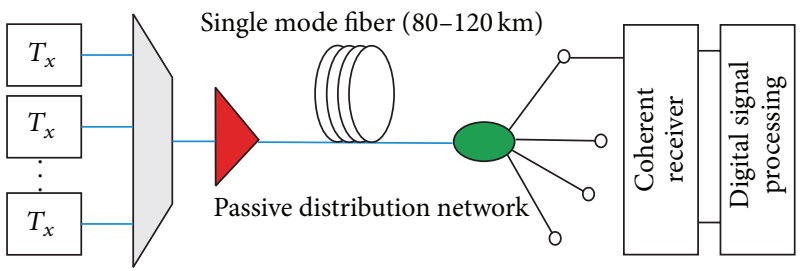

FIGURE 3: Architecture of coherent $10 \mathrm{Gbit} / \mathrm{s}$ long haul WDM-PON employing DP-QPSK downstream signals.

to transmit over a distance of $120 \mathrm{~km}$. The physical parameters of SMF fiber are attenuation $\alpha=+0.2 \mathrm{~dB} / \mathrm{km}$, dispersion $D=+16.75 \mathrm{ps} /(\mathrm{nm}-\mathrm{km})$, and nonlinear coefficient $\gamma=$ $+1.3\left(\mathrm{~km}^{-1} \cdot \mathrm{W}^{-1}\right)$. No midspan repeaters are used in the transmission link. For our investigations, all the ONUs are considered at the same transmission distance and having the same power budget.

The signal is detected with a phase and polarization diverse coherent receiver. At the receiver, the channels can be selected through the tunable local oscillator (LO) laser source. The transmission performance of the middle channel of the WDM grid is monitored and is quantified by the biterror-ratio (BER). The FEC threshold limit corresponds to the BER level of $3.8 \times 10^{-3}$ (as indicated by the dashed line in graphs). As we are investigating the deterministic impairments (linear and nonlinear effects) of fiber transmission, we have considered the effect of polarization mode dispersion (PMD) and laser line width negligible in these numerical analyses [8]. The model is implemented by using OptiSystem v.12 and DSP modules are implemented in Matlab. 


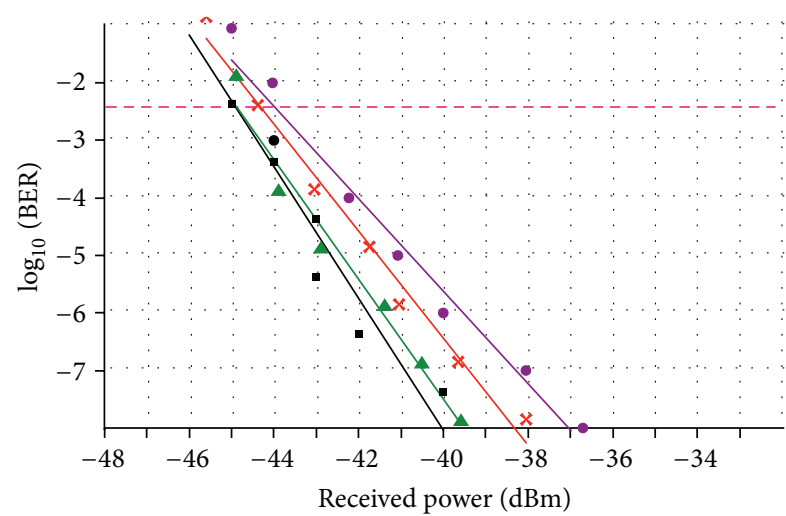

$\begin{array}{ll}\text { Back-to-back } & \\ -1 \text { channel } & -10 \mathrm{GHz} \text { spacing } \\ -50 \mathrm{GHz} \text { spacing } & -5 \mathrm{GHz} \text { spacing }\end{array}$

FIGURE 4: Back-to-back sensitivity measurements for single channel and WDM transmission with different channel spacings.

\section{Results and Discussion}

Figure 4 shows the back-to-back receiver sensitivity and the impact of channel spacing on WDM-PON. At $50 \mathrm{GHz}$ channel spacing, there is no significant nonlinear penalty observed at the FEC threshold limit. As the narrow channel spacing is used, that is, $10 \mathrm{GHz}$ and $5 \mathrm{GHz}$, we have observed a penalty of $0.75 \mathrm{~dB}$ and $1.1 \mathrm{~dB}$, respectively, at a BER level of $3.8 \times 10^{-3}$. The results depict that, for long haul transmission in PONs, nonlinearities are the major degrading factors with narrow channel spacing. We have further investigated the impact of nonlinearities and their compensation on the receiver sensitivity. As narrow channel spacing, that is, $5 \mathrm{GHz}$ channel spaced transmission, has dominant multichannel nonlinear impairments, that is, cross-phase modulation (XPM), we have investigated this scenario for different signal launch powers and resultant impact of using NL equalization techniques.

Figure 5 shows the performance of $5 \mathrm{GHz}$ channel spaced WDM-PON transmission over $120 \mathrm{~km}$ SMF link. At $3 \mathrm{dBm}$ signal launch power, the incurred penalty is less than $2 \mathrm{~dB}$. The NL equalization methods, that is, BP and VE, show efficient and improved transmission performance. The incurred penalty is reduced to $1.51 \mathrm{~dB}$ with the postprocessing of data by $\mathrm{BP}$ and VE. While we have not observed any prominent improvement in system performance by employing OPC$\mathrm{NM}$ around the region of $3.8 \times 10^{-3} \mathrm{BER}$, at higher launch power, that is, $6 \mathrm{dBm}$, nonlinear impairments dominate and the system performance is degraded, as in Figure 6. We have observed a penalty of $4 \mathrm{~dB}$ with respect to back-to-back transmission. The results of NL equalization show significant improvement in performance reducing the penalty to $2 \mathrm{~dB}$ by $\mathrm{BP}$ algorithm, $1.5 \mathrm{~dB}$ by VE algorithm, and $2.69 \mathrm{~dB}$ by OPCNM technique. By virtue of this performance, the system can be operated at higher transmission powers and physical transmission distance for the ONUs can be increased.

Furthermore, we have investigated the number of ONUs (network subscribers) for maximum split ratio, that is,

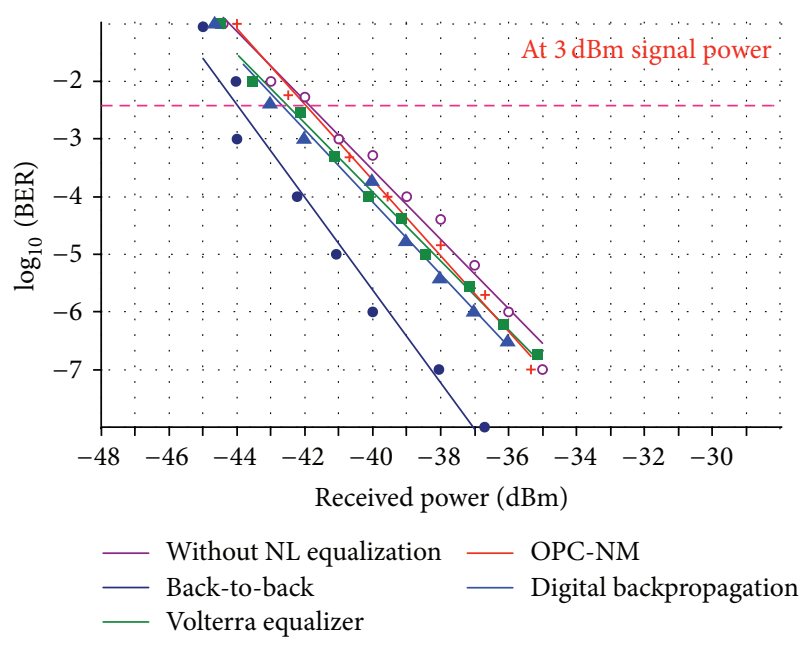

FIgure 5: Performance analysis at $5 \mathrm{GHz}$ channel spacing for $3 \mathrm{dBm}$ signal launch power using BP, VE, and OPC-NM.

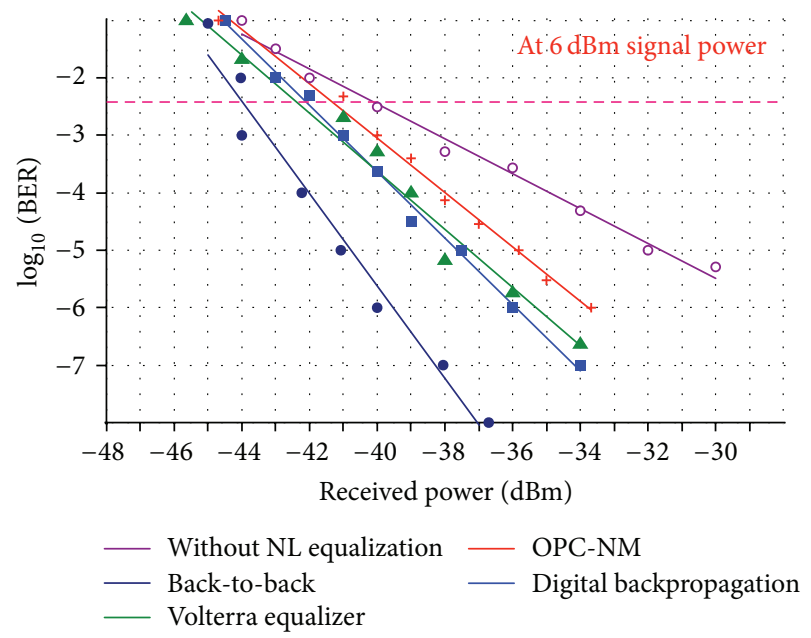

FIGURE 6: Performance analysis at $5 \mathrm{GHz}$ channel spacing for $6 \mathrm{dBm}$ signal launch power using BP, VE, and OPC-NM.

$1: 1024$, with respect to obtainable transmission distance, as in Figure 7. As we are emphasizing on the nonlinear compensation, we plotted the graph for the system with $5 \mathrm{GHz}$ channel spacing at $6 \mathrm{dBm}$ signal launch power. The maximum transmission distance for $5 \mathrm{GHz}$ channel spaced system after a 1:1024 split ratio is found to be $92.06 \mathrm{~km}$, whereas, by employing VE algorithm, we are able to get the transmission distance of $126.6 \mathrm{~km}$ for the same split ratio.

\section{Conclusion}

To summarize, special focus is given to compensate fiber nonlinear transmission impairments and their interplay between transmission distance and split ratio. We have numerically evaluated the nonlinear equalization algorithms in $10 \mathrm{Gbit} / \mathrm{s}$ DP-QPSK long haul WDM-PON over $120 \mathrm{~km}$ fiber link without midspan repeaters. The results of NL equalization, 


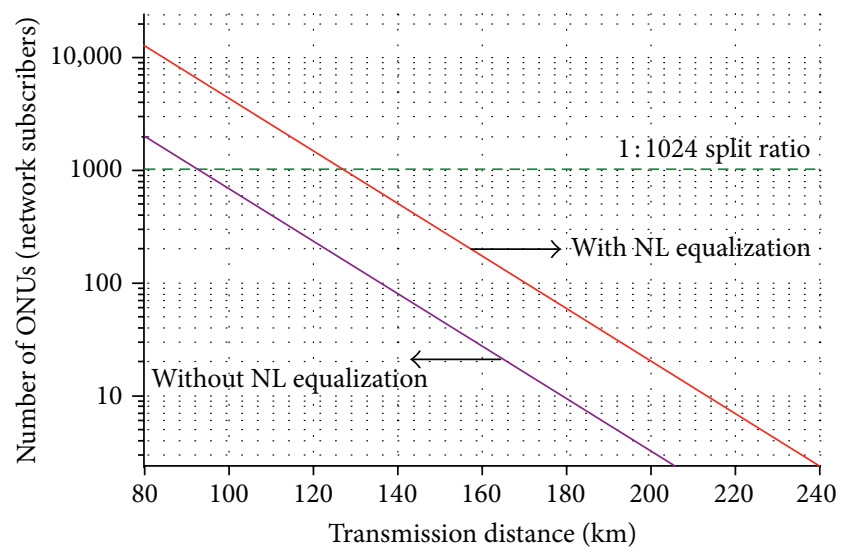

FIgURE 7: Performance analysis for number of ONUs (network subscribers) versus maximum transmission distance.

with $5 \mathrm{GHz}$ channel spacing and at $6 \mathrm{dBm}$ signal launch power, show significant improvement in system performance reducing the penalty to $2 \mathrm{~dB}$ by $\mathrm{BP}$ algorithm, $1.5 \mathrm{~dB}$ by VE algorithm, and $2.69 \mathrm{~dB}$ by OPC-NM technique at the BER level of $3.8 \times 10^{-3}$.

Moreover, the maximum transmission distance after a $1: 1024$ split ratio is improved by $35.4 \mathrm{~km}$ by VE algorithm, which is the optimal NL equalization technique. From the over-all results, we conclude that NL equalization techniques are beneficial for PONs. They compensate the impairments to a considerable extent and will be helpful in the next generation networks where higher-order modulation formats, that is, m-ary QAM, will be implemented at access network level.

\section{Conflict of Interests}

The authors declare that there is no conflict of interests regarding the publication of this paper.

\section{Acknowledgments}

The authors would like to thank Professor Dr.-Ing. Bernhard Schmauss and Dr.-Ing. Chien-Yu Lin for their everlasting support during the research tenure at University of ErlangenNuremberg, Germany.

\section{References}

[1] D. Lavery, M. Ionescu, S. Makovejs, E. Torrengo, and S. J. Savory, "A long-reach ultra-dense $10 \mathrm{Gbit} / \mathrm{s}$ WDM-PON using a digital coherent receiver," Optics Express, vol. 18, no. 25, pp. 2585525860, 2010.

[2] X. Li, X. Chen, G. Goldfarb et al., "Electronic post-compensation of WDM transmission impairments using coherent detection and digital signal processing," Optics Express, vol. 16, no. 2, pp. 880-888, 2008.

[3] J. D. Reis, D. M. Neves, and A. L. Teixeira, "Analysis of nonlinearities on coherent ultradense WDM-PONs using Volterra series," Journal of Lightwave Technology, vol. 30, no. 2, Article ID 6112703, pp. 234-241, 2012.
[4] W. Rosenkranz and J. von Hoyningen-Huene, "Nonlinearity compensation and equalization in access networks," in Proceedings of the 17th Opto-Electronics and Communications Conference (OECC '12), pp. 459-460, Busan, Republic of Korea, July 2012.

[5] E. Ip and J. M. Kahn, "Compensation of dispersion and nonlinear impairments using digital backpropagation," IEEE Journal of Lightwave Technology, vol. 26, no. 20, pp. 3416-3425, 2008.

[6] D. S. Millar, S. Makovejs, C. Behrens et al., "Mitigation of fiber nonlinearity using a digital coherent receiver," IEEE Journal on Selected Topics in Quantum Electronics, vol. 16, no. 5, pp. 12171226, 2010.

[7] R. Asif, C. Lin, M. Holtmannspoetter, and B. Schmauss, "Multispan digital non-linear compensation for dual-polarization quadrature phase shift keying long-haul communication systems," Optics Communications, vol. 285, no. 7, pp. 1814-1818, 2012.

[8] R. Asif, C. Lin, and B. Schmauss, "Impact of channel baudrate on logarithmic digital backward propagation in DP-QPSK system with uncompensated transmission links," Optics Communications, vol. 284, no. 24, pp. 5673-5677, 2011.

[9] R. Asif, C. Y. Lin, M. Holtmannspoetter, and B. Schmauss, "Optimized digital backward propagation for phase modulated signals in mixed-optical fiber transmission link," Optics Express, vol. 18, no. 22, pp. 22796-22807, 2010.

[10] R. Asif, C. Lin, M. Holtmannspoetter, and B. Schmauss, "Lowcomplexity logarithmic step-size-based filtered digital backward propagation algorithm for compensating fiber transmission impairments," in Next-Generation Optical Communication: Components, Sub-Systems, and Systems, vol. 8242 of Proceedings of SPIE, San Francisco, Calif, USA, January 2012.

[11] R. Asif, M. Usman, C. Lin, and B. Schmauss, "Application of a digital non-linear compensation algorithm for evaluating the performance of root-raised-cosine pulses in $112 \mathrm{Gbits}^{-1} \mathrm{DP}$ QPSK transmission," Journal of Optics, vol. 14, no. 9, Article ID 095402, 2012.

[12] R. Asif, C. Y. Lin, and B. Schmauss, "Logarithmic versus modified digital backward propagation algorithm in $224 \mathrm{~Gb} / \mathrm{s}$ DP-16QAM transmission over dispersion uncompensated fiber links," Optical Engineering, vol. 51, no. 4, Article ID 045007, 2012.

[13] H. Chin, M. Forzati, and J. Mrtensson, "Volterra based nonlinear compensation on $224 \mathrm{~Gb} / \mathrm{s}$ PolMux-16QAM optical fibre link," in Proceedings of the Optical Fiber Communication Conference, OSA Technical Digest, Optical Society of America, paper JW2A.61, 2012.

[14] T. Freckmann and J. Speidel, "Linear and nonlinear electronic feed-forward equalizers for DQPSK," in Proceedings of the 20th Annual Meeting of the IEEE Lasers and Electro-Optics Society (LEOS '07), Paper MP4, pp. 145-146, October 2007.

[15] M. D. Pelusi and B. J. Eggleton, "Tunable all-optical precompensation of fiber Kerr effect on multi-format DPSK signals using a nonlinearity module," in Proceedings of the Optical Fiber Communication Conference and Exposition and the National Fiber Optic Engineers Conference (OFC/NFOEC '12), pp. 1-3, March 2012.

[16] R. Asif, H. Shahid, F. Arshad, and R. Saleem, "Scalable nonlinear equalization in high-bit-rate optical transmission systems," Photonics Research, vol. 1, p. 135, 2013. 

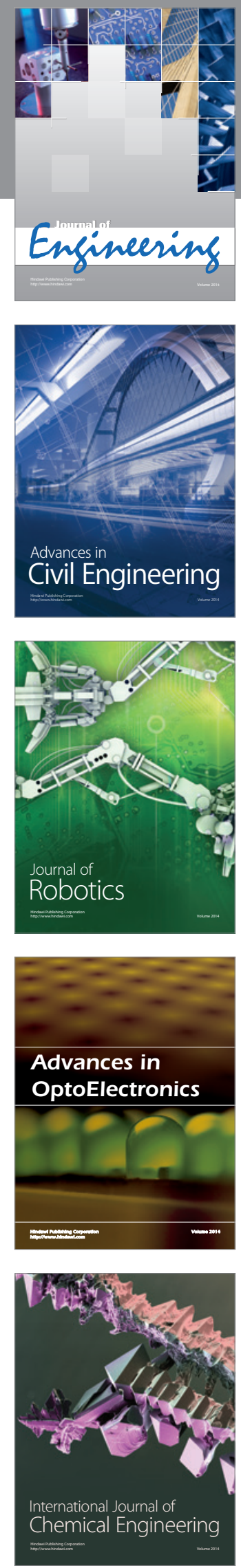

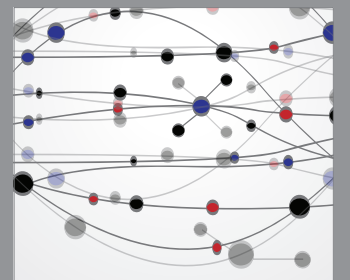

The Scientific World Journal
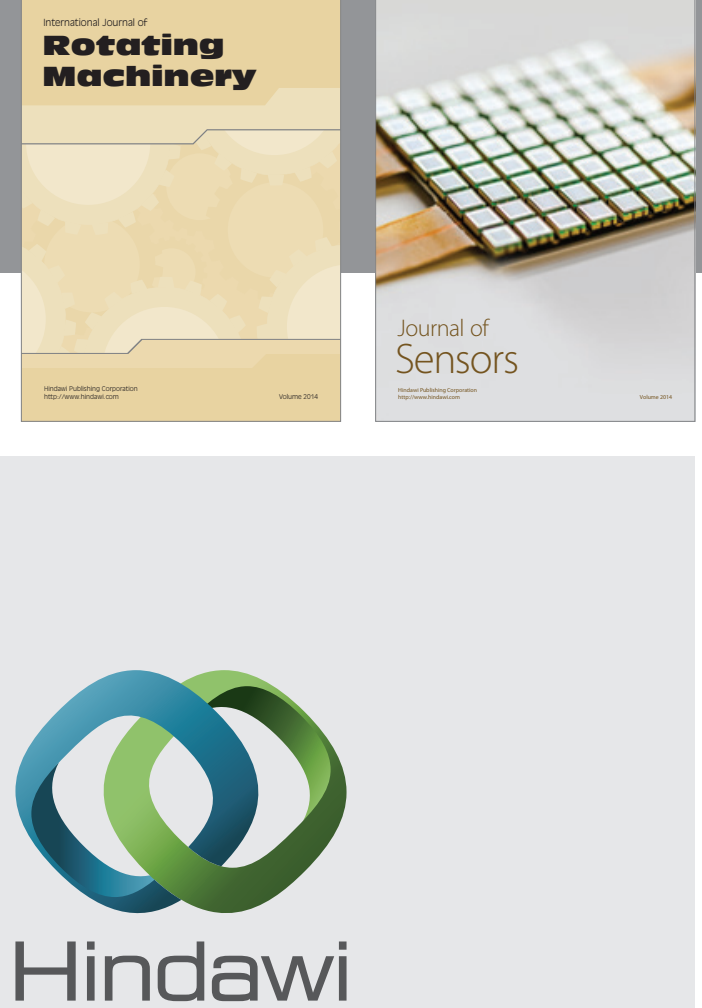

Submit your manuscripts at http://www.hindawi.com
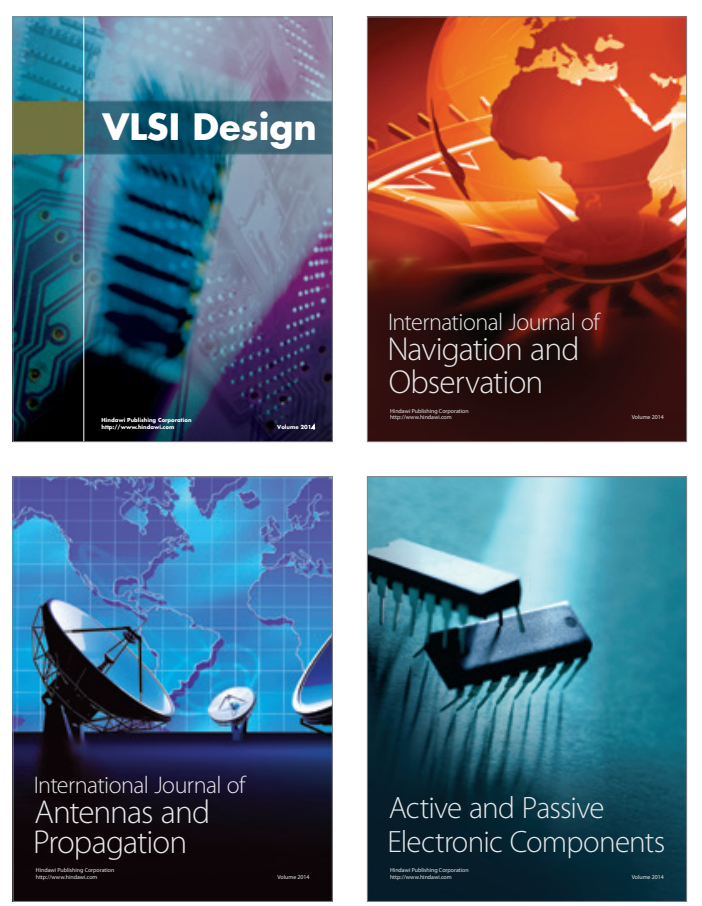
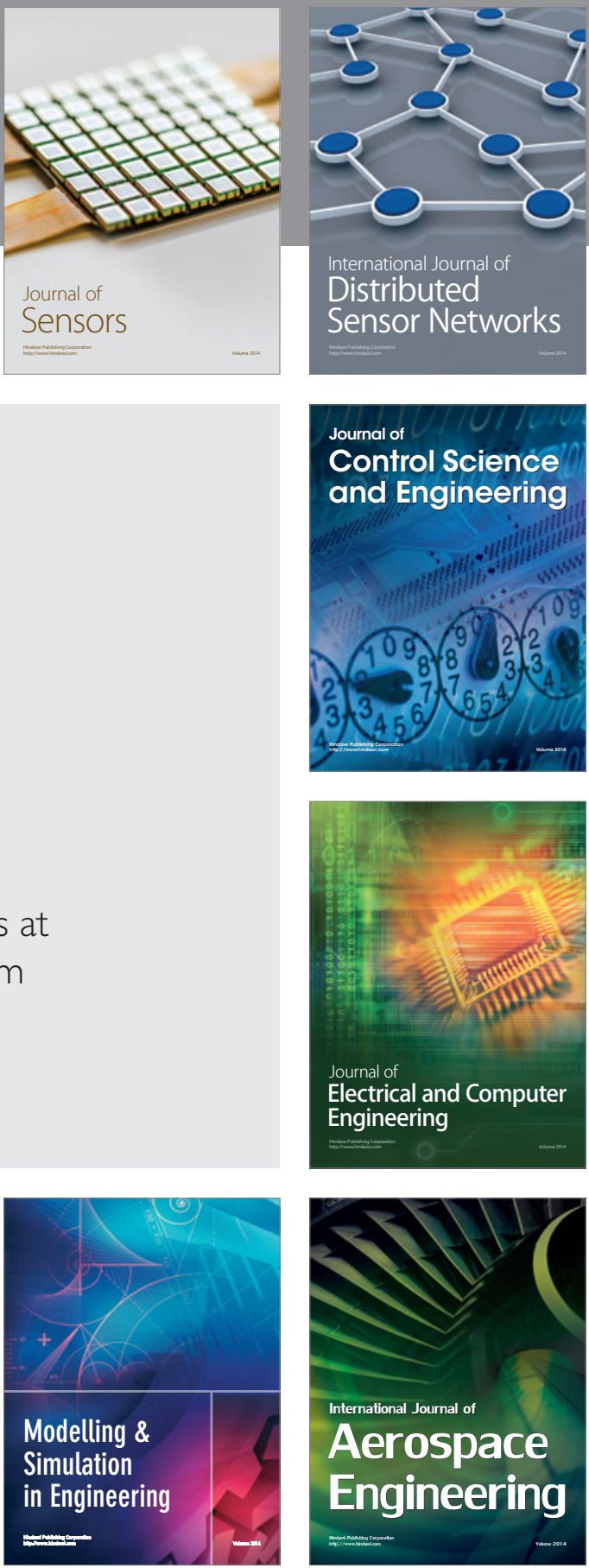

Journal of

Control Science

and Engineering
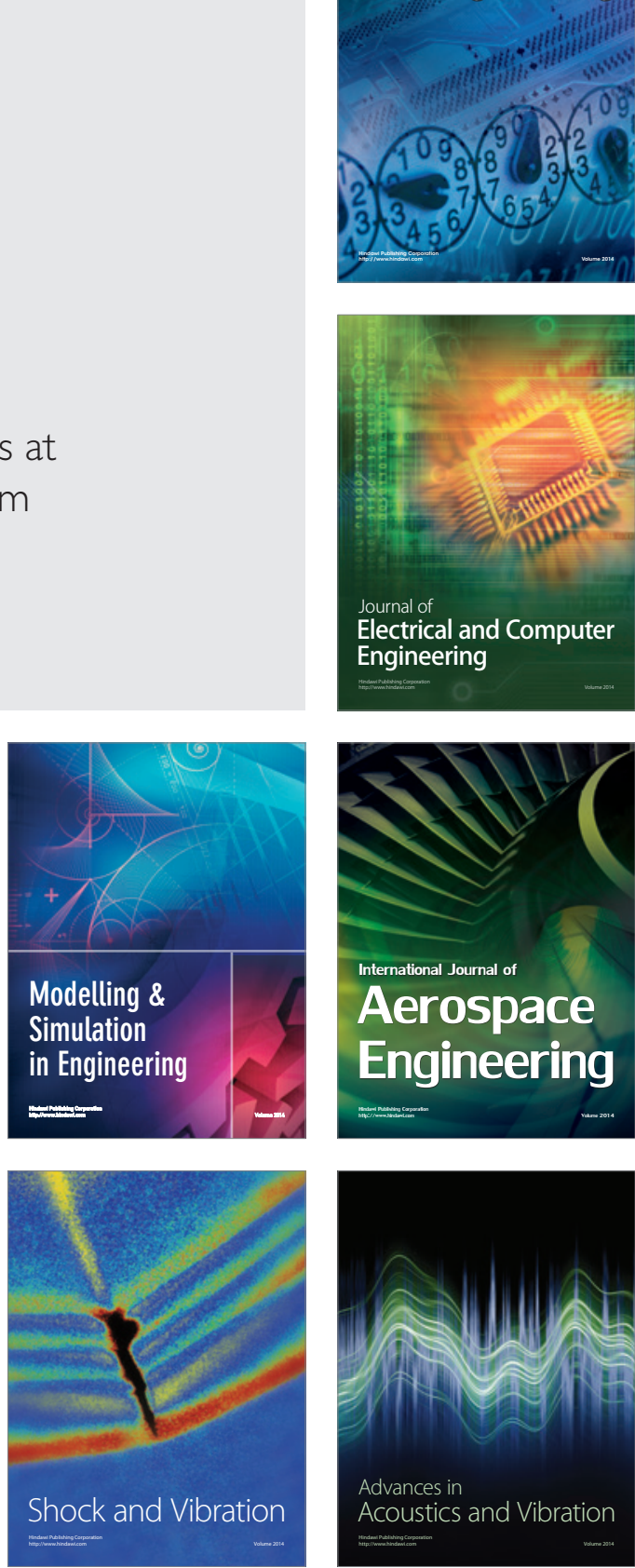